PROCEEDINGS OF THE

AMERICAN MATHEMATICAL SOCIETY

Volume 125, Number 5, May 1997, Pages 1435-1441

S 0002-9939(97)03623-X

\title{
ON POWER BOUNDED OPERATORS
}

EUGEN J. IONASCU

(Communicated by Palle E. T. Jorgensen)

\begin{abstract}
In this paper we generalize the following consequence of a wellknown result of Nagy: if $T$ and $T^{-1}$ are power bounded operators, then $T$ is a polynomially bounded operator.
\end{abstract}

Let $\mathcal{H}$ be a separable, infinite dimensional, complex Hilbert space, and let $\mathcal{L}(\mathcal{H})$ denote the algebra of all bounded linear operators on $\mathcal{H}$. Recall that an operator $T \in \mathcal{L}(\mathcal{H})$ is called power bounded (notation: $T \in \mathcal{P} \mathcal{W}(\mathcal{H})$ ) if there exists a constant $M(\geq 1)$ such that

$$
\left\|T^{n}\right\| \leq M, \quad n \in \mathbb{N},
$$

and $T$ is called polynomially bounded (notation: $T \in \mathcal{P B}(\mathcal{H})$ ) if there exists a constant $M(\geq 1)$ such that

$$
\|p(T)\| \leq M\|p\|_{\infty}
$$

for every polynomial $p$, where $\|p\|_{\infty}=\sup \{|p(z)|: z \in \mathbb{C}, \quad|z| \leq 1\}$. The smallest number $M$ satisfying (1) (resp., (2)) is called the power bound (resp., the polynomial bound ) of $T$ and will be denoted by $M_{w}(T)$ (resp., $M_{p}(T)$ ), or simply $M_{w}$ (resp., $M_{p}$ ) when no confusion is possible. One knows (cf. $[1,2,4]$ ) that $\mathcal{P} \mathcal{W}(\mathcal{H})$ strictly contains the class $\mathcal{P B}(\mathcal{H})$, but there is a theorem of Nagy [3] which says that every $T \in \mathcal{P W}(\mathcal{H})$ such that $T^{-1}$ exists and belongs to $\mathcal{P} \mathcal{W}(\mathcal{H})$ is similar to a unitary operator, and therefore is polynomially bounded. The purpose of this note is to establish the following two stronger results than the above-mentioned consequence of Nagy's theorem.

Theorem 1.1. Suppose $T \in P \mathcal{W}(\mathcal{H})\left(\right.$ with $\left.M_{w}(T)>1\right)$ and the following inequality holds for some positive number $\alpha$ and a strictly increasing sequence $\left\{n_{k}\right\} \subset \mathbb{N}$ :

$$
1 / n_{k} \sum_{j=0}^{n_{k}} T^{* j} T^{j} \geq \alpha\left(I-P_{k e r(T)}\right),
$$

where $P_{\text {ker }(T)}$ is the (orthogonal) projection on the kernel of $T$. Then $T \in \mathcal{P B}(\mathcal{H})$ and the polynomial bound $M_{p}$ of $T$ satisfies

$$
M_{p} \leq M_{w}(T)^{3}\left(\frac{M_{w}(T)^{2}-1}{\alpha \ln M_{w}(T)}\right)^{1 / 2}+1 .
$$

Received by the editors June 19, 1995 and, in revised form, November 20, 1995.

1991 Mathematics Subject Classification. Primary 47B99.

(C)1997 American Mathematical Society 
Theorem 1.2. Suppose $T \in \mathcal{P} \mathcal{W}(\mathcal{H})$ and the following inequality holds for some positive number $\alpha$ and a strictly increasing sequence $\left\{t_{k}\right\}_{k \in \mathbb{N}}$ of real numbers converging to 1 :

$$
\left(1-t_{k}\right) \sum_{j=0}^{\infty} t_{k}^{j} T^{* j} T^{j} \geq \alpha\left(I-P_{k e r(T)}\right)
$$

Then $T \in \mathcal{P B}(\mathcal{H})$, and the polynomial bound $M_{p}$ of $T$ satisfies

$$
M_{p} \leq\left(\frac{14}{\alpha}\right)^{1 / 2} M_{w}^{3}
$$

As mentioned above, the following is an immediate consequence of either Theorem 1.1 or Theorem 1.2 .

Corollary 1.3 (Nagy [4]). If $T \in \mathcal{P W}(\mathcal{H})$ is invertible and $T^{-1} \in \mathcal{P} \mathcal{W}(\mathcal{H})$, then $T$ is polynomially bounded.

In order to prove Theorem 1.1, we use the following lemma, which is well known [5].

Lemma 1.4. Suppose $S \in \mathcal{L}(\mathcal{H})$ is such that $S^{m}$ is a contraction for some integer $m \geq 2$. Then $S$ is similar to a contraction, and, in particular,

$$
A=\left(I+S^{*} S+\ldots+S^{*(m-1)} S^{(m-1)}\right)^{1 / 2}
$$

is an invertible operator that satisfies

$$
\left\|A S A^{-1}\right\| \leq 1
$$

Proof. Clearly $A$ is an invertible selfadjoint operator. To establish (8) it is enough to check that

$$
\left\|A S A^{-1} h\right\| \leq\|h\| \quad, \quad h \in \mathcal{H} .
$$

For a given $h$, define $g=A^{-1} h$, and hence (9) becomes equivalent to

$$
\left\langle A^{2} S g, S g\right\rangle \leq\left\langle A^{2} g, g\right\rangle \text {. }
$$

Using (7), we see that (10) is equivalent to

$$
\sum_{j=1}^{m}\left\|S^{j} g\right\|^{2} \leq \sum_{j=0}^{m-1}\left\|S^{j} g\right\|^{2},
$$

which is true since $\left\|S^{m} g\right\| \leq\|g\|$.

Proof of Theorem 1.1. For brevity we write $M=M_{w}(T)>1$. For each $n \in \mathbb{N}$, set $\alpha_{n}=M^{1 / n}, \beta_{n}=\alpha_{n}^{-1}$, and note that $\beta_{n}<1<\alpha_{n}$. Since $\left\|\left(\beta_{n} T\right)^{n}\right\| \leq 1$ for each $n \in \mathbb{N}$, we may apply Lemma 1.4 to obtain for each $n \in \mathbb{N}$ a contraction $C_{n}$ such that

$$
\beta_{n} T=A_{n}^{-1} C_{n} A_{n}
$$

where $A_{n}=\left(\sum_{j=0}^{n-1} \beta_{n}^{2 j} T^{* j} T^{j}\right)^{1 / 2}$. Consider now an arbitrary polynomial $p(z)=$ $a_{0}+a_{1} z+a_{2} z^{2}+\ldots+a_{l} z^{l}$. Then

$$
p(T)=p\left(\alpha_{n} A_{n}^{-1} C_{n} A_{n}\right)=A_{n}^{-1} p\left(\alpha_{n} C_{n}\right) A_{n} .
$$


Applying the von Neumann inequality to $C_{n}$ and the polynomial $q_{n}(z)=p\left(\alpha_{n} z\right)$, we conclude from (12) that

$$
\|p(T)\| \leq\left\|A_{n}^{-1}\right\|\left\|A_{n}\right\|\left\|q_{n}\right\|_{\infty}, \quad n \in \mathbb{N} .
$$

Let us observe now that for each $n \in \mathbb{N}$,

$$
\left\|A_{n}\right\|^{2}=\left\|A_{n}^{2}\right\| \leq \sum_{j=0}^{n-1} \beta_{n}^{2 j} M^{2}=M^{2}\left(1-\beta_{n}^{2 n}\right) /\left(1-\beta_{n}^{2}\right)=M^{2}\left(1-M^{-2}\right) /\left(1-\beta_{n}^{2}\right),
$$

so

$$
\left\|A_{n}\right\| \leq\left(M^{2}-1\right)^{1 / 2} /\left(1-\beta_{n}^{2}\right)^{1 / 2} .
$$

Moreover, for each $n \in \mathbb{N},\left\|A_{n}^{-1}\right\|=\gamma\left(A_{n}\right)^{-1}$, where $\gamma\left(A_{n}\right)$ is the greatest number $\gamma>0$ with the property that $\left\|A_{n} h\right\| \geq \gamma\|h\|$ for all $h \in \mathcal{H}$. Equivalently,

$$
\left\langle A_{n}^{2} h, h\right\rangle \geq \gamma\left(A_{n}\right)^{2}\langle h, h\rangle, h \in \mathcal{H}, \quad n \in \mathbb{N} .
$$

Consider now the case that $\operatorname{ker}(T)=\{0\}$. Let $\left\{n_{k}\right\}$ be the sequence from (3). Then

$$
A_{n_{k}}^{2}=\sum_{j=0}^{n_{k}-1} \beta_{n_{k}}^{2 j} T^{* j} T^{j} \geq \beta_{n_{k}}^{2 n_{k}} \sum_{j=0}^{n_{k}-1} T^{* j} T^{j} \geq \beta_{n_{k}}^{2 n_{k}} n_{k} \alpha I=n_{k} M^{-2} \alpha I, \quad k \in \mathbb{N} .
$$

Therefore, $\gamma\left(A_{n_{k}}\right) \geq n_{k}^{1 / 2} M^{-1} \alpha^{1 / 2}$ for each $k \in \mathbb{N}$, which implies that

$$
\left\|A_{n_{k}}^{-1}\right\|=\gamma\left(A_{n_{k}}\right)^{-1} \leq n_{k}^{-1 / 2} M \alpha^{-1 / 2} .
$$

Thus, from (14) and (15) we get

$$
\left\|A_{n_{k}}\right\|\left\|A_{n_{k}}^{-1}\right\| \leq M\left(M^{2}-1\right)^{1 / 2} \alpha^{-1 / 2} /\left(n_{k}\left(1-\beta_{n_{k}}^{2}\right)\right)^{1 / 2}, k \in \mathbb{N} .
$$

A simple continuity argument shows that for $p$ fixed we have

$$
\lim _{k \rightarrow \infty}\left\|q_{n_{k}}\right\|_{\infty}=\|p\|_{\infty} \text {. }
$$

Going back to (13), and taking into account (16) and (17), we can let $k$ go to infinity and obtain the inequality

$$
\|p(T)\| \leq M\left(\left(M^{2}-1\right) / 2 \ln M\right)^{1 / 2} \alpha^{-1 / 2}\|p\|_{\infty}
$$

by using the formula (from elementary calculus)

$$
\lim _{n \rightarrow \infty}\left(M^{2 / n}-1\right) n=2 \ln M .
$$

Thus, in this case, $T \in \mathcal{P B}(\mathcal{H})$ and (4) is valid.

Let us consider now the general case. With respect to the decomposition $\mathcal{H}=$ $($ ker $T) \oplus(\operatorname{ker} T)^{\perp}, T$ has an operator matrix

$$
T=\left[\begin{array}{ll}
0 & S \\
0 & Q
\end{array}\right]
$$

where $S:(\operatorname{ker} T)^{\perp} \rightarrow(\operatorname{ker} T)$ is a bounded linear operator, $Q \in \mathcal{P} \mathcal{W}\left((\operatorname{ker} T)^{\perp}\right)$, and $M_{w}(Q) \leq M_{w}(T)$. For each polynomial $p$ one sees easily that

$$
p(T)=\left[\begin{array}{cc}
p(0) I & S q(Q) \\
0 & p(Q)
\end{array}\right]
$$


where $q(z)=(p(z)-p(0)) / z$. Therefore, since $\|q\|_{\infty} \leq 2\|p\|_{\infty}$, it is sufficient to show that $Q$ is polynomially bounded and has an appropriate polynomial bound. We want to use the first case, so let us observe that

$$
T^{* k} T^{k} \leq\left[\begin{array}{cc}
0 & 0 \\
0 & \left(\|S\|^{2}+\|Q\|^{2}\right) Q^{* k-1} Q^{k-1}
\end{array}\right], k \in \mathbb{N} .
$$

But (3) and (20) together yield

$$
\left(\|S\|^{2}+\|Q\|^{2}\right) /\left(n_{k}-1\right) \sum_{j=0}^{n_{k}-1} Q^{* j} Q^{j} \geq\left(\alpha-(\alpha+1) /\left(n_{k}-1\right)\right) I_{(k e r T)^{\perp}} .
$$

In particular this says that if $h \in \operatorname{ker}(Q) \cap(\operatorname{ker} T)^{\perp}$, then

$$
\left(\|S\|^{2}+\|Q\|^{2}\right) /\left(n_{k}-1\right)\langle h, h\rangle \geq\left(\alpha-(\alpha+1) /\left(n_{k}-1\right)\right)\langle h, h\rangle,
$$

and letting $k$ go to infinity we obtain that $h=0$. Hence, $Q$ satisfies the condition (3) in the case when $\operatorname{ker}(Q)=\{0\}$ for $\alpha^{\prime}=(\alpha-\epsilon) /\left(\|S\|^{2}+\|Q\|^{2}\right)>0$ and a subsequence $\left\{n_{k}-1\right\}$ for $k$ large enough (depending upon $\epsilon$ ). Therefore, we obtain from the previous case,

$$
\|p(Q)\| \leq M\left(\left(M^{2}-1\right) / 2 \ln M\right)^{1 / 2} \alpha^{-1 / 2}\left(\|S\|^{2}+\|Q\|^{2}\right)^{1 / 2}\|p\|_{\infty},
$$

since $\epsilon>0$ was arbitrary. Finally we get

$$
\begin{aligned}
\|p(T)\| & \leq\left(M\left(\left(M^{2}-1\right) / 2 \ln M\right)^{1 / 2} \alpha^{-1 / 2}\left(\|S\|^{2}+\|Q\|^{2}\right)^{1 / 2}\|S\|+1\right)\|p\|_{\infty} \\
& \leq\left(M^{3}\left(\left(M^{2}-1\right) / \ln M\right)^{1 / 2} \alpha^{-1 / 2}+1\right)\|p\|_{\infty},
\end{aligned}
$$

which is what we wanted to show.

We want to consider now the continuous analog of Theorem 1.1.

Proof of Theorem 1.2. Let us define for $T \in \mathcal{P W}(\mathcal{H})$ and every $t \in[0,1)$ the self-adjoint invertible operator

$$
A_{t}=(1-t)^{1 / 2}\left(\sum_{j=0}^{\infty} t^{j} T^{* j} T^{j}\right)^{1 / 2} .
$$

First, observe that this operator is well-defined for $T \in \mathcal{P} \mathcal{W}(\mathcal{H})$, and moreover

$$
\left\|A_{t}\right\|^{2}=\left\|A_{t}^{2}\right\| \leq(1-t) \sum_{j=0}^{\infty} t^{j}\left\|T^{j}\right\|\left\|T^{* j}\right\| \leq M_{w}(T)^{2} .
$$

As before, let us consider the case when $\operatorname{ker}(T)=\{0\}$. If (5) is satisfied, then $\left\|A_{t}^{-1}\right\| \leq \alpha^{-1 / 2}$ at least for $t=t_{k}$.

Now observe that for any $h \in \mathcal{H}$ we have

$$
(1-t)\left\|A_{t}^{-1} h\right\|^{2}+t\left\|A_{t} T A_{t}^{-1} h\right\|^{2}=\|h\|^{2},
$$

which, in particular, says that $t^{1 / 2} A_{t} T A_{t}^{-1}$ is a contraction. Hence we can use the idea from the proof of Theorem 1.1 to get that

$$
\|p(T)\| \leq\left\|A_{t_{k}}\right\|\left\|A_{t_{k}}^{-1}\right\|\left\|q_{k}\right\|_{\infty}, \quad k \in \mathbb{N}
$$

where $q_{k}(z)=p\left(t_{k}^{-1 / 2} z\right)$ for any given polynomial $p$. Letting $k$ go to infinity we get the inequality

$$
\|p(T)\| \leq M_{w}(T) \alpha^{-1 / 2}\|p\|_{\infty},
$$


which is what we wanted to show in the case $\operatorname{ker}(T)=0$. In the general case, if $T$ has the decomposition (19), by using the inequality (20) and the hypothesis (5), we have that

$$
\left(\|S\|^{2}+\|Q\|^{2}\right)\left(1-t_{k}\right) \sum_{j=1}^{\infty} t_{k}^{j} Q^{* j-1} Q^{j-1} \geq\left(\alpha-1+t_{k}\right) I_{(\text {ker } T)^{\perp}},
$$

which says, first, that $\operatorname{ker}(Q)=0$ and thus that $Q$ is as in the first case. Therefore. we finally get

$$
\begin{aligned}
\|p(T)\| & \leq M_{w}(T)\left\{\left(3+4\|S\|^{2}\right)\left(\frac{\|S\|^{2}+\|Q\|^{2}}{\alpha}\right)\right\}^{1 / 2}\|p\|_{\infty} \\
& \leq\left(\frac{14}{\alpha}\right)^{1 / 2} M_{w}(T)^{3}\|p\|_{\infty},
\end{aligned}
$$

which was to be proved.

An easy corollary of Theorem 1.2 is the following generalization.

Corollary 1.5. Suppose $T \in \mathcal{P} \mathcal{W}(\mathcal{H})$ and the following inequality holds for some $n \in \mathbb{N}$, some positive number $\alpha$, and a strictly increasing sequence $\left\{t_{k}\right\}_{k \in \mathbb{N}}$ of real numbers converging to 1 :

$$
\left(1-t_{k}\right) \sum_{j=0}^{\infty} t_{k}^{j} T^{* j} T^{j} \geq \alpha\left(I-P_{k e r\left(T^{n}\right)}\right) .
$$

Then $T$ is polynomially bounded.

Proof. With respect to the decomposition $\mathcal{H}=\operatorname{ker}\left(T^{n}\right) \oplus\left(\operatorname{ker}\left(T^{n}\right)\right)^{\perp}, T$ has the operator matrix

$$
T=\left[\begin{array}{ll}
A & B \\
C & D
\end{array}\right]
$$

Since $\operatorname{ker}\left(T^{n}\right)$ is an invariant subspace for $T$, the operator $C$ must be zero. In addition, we have that

$$
T^{n}=\left[\begin{array}{ll}
0 & E \\
0 & F
\end{array}\right]
$$

where

$$
A^{n}=0, \quad F=D^{n}, \quad E=\sum_{j=0}^{j=n} A^{j} B D^{n-j} .
$$

Now, for an arbitrary operator $T, T \in \mathcal{P B}(\mathcal{H})$ if and only if $T^{m} \in \mathcal{P B}(\mathcal{H})$ for some $m \in \mathbb{N}$. This can be easily seen if we observe that for any polynomial $p$, there exists a unique decomposition of the form

$$
p(z)=p_{1}(z)+z p_{2}(z)+z^{2} p_{3}(z)+\ldots+z^{m-1} p_{m}(z),
$$


where $p_{1}, p_{2}, p_{3}, \ldots, p_{m}$ are polynomials in $z^{m}$ and $\left\|p_{j}\right\|_{\infty} \leq\|p\|_{\infty}$ for $j=$ $1,2, \ldots, m$. Hence, it suffices to show that $F$ and therefore $D$ is polynomially bounded. But now, since for any integer $k \geq 0$

$$
\left(I-P_{k e r\left(T^{n}\right)}\right) T^{* k} T^{k}\left(I-P_{k e r\left(T^{n}\right)}\right)=\left[\begin{array}{cc}
0 & 0 \\
0 & D^{* k} D^{k}
\end{array}\right],
$$

which follows from (23) multiplying from the left and from the right by $I-P_{k e r\left(T^{n}\right)}$, we obtain that $D$ satisfies the hypothesis of Theorem 1.1. This means that $D$ is polynomially bounded, and so $F$ and $T$ are also.

Comments. If we start with a contraction $T$, let us show that the function $A_{t}$ defined in (22) satisfies $A_{t} \geq A_{s}$ for $0 \leq t<s \leq 1$. Indeed, since $A^{2} \geq B^{2}$ for positive simidefinite operators implies $A \geq B$, it is enough to check that $A_{t}^{2} \geq A_{s}^{2}$ $(t<s)$. This is equivalent to

$$
(1-t) \sum_{j=0}^{\infty} t^{j}\left\|T^{j} h\right\|^{2} \geq(1-s) \sum_{j=0}^{\infty} s^{j}\left\|T^{j} h\right\|^{2}, h \in \mathcal{H},
$$

and this can be written in the following equivalent form which is clearly true for $T$ a contraction:

$$
\begin{aligned}
& (s-t)\left(\|h\|^{2}-\|T h\|^{2}\right)+\left(s^{2}-t^{2}\right)\left(\|T h\|^{2}-\left\|T^{2} h\right\|^{2}\right) \\
& \quad+\left(s^{3}-t^{3}\right)\left(\left\|T^{2} h\right\|^{2}-\left\|T^{3} h\right\|^{2}\right)+\left(s^{4}-t^{4}\right)\left(\left\|T^{3} h\right\|^{2}-\left\|T^{4} h\right\|^{2}\right)+\ldots \geq 0 .
\end{aligned}
$$

Therefore, it is interesting to ask: what is the class of operators for which the function $t \rightarrow A_{t}$ is decreasing? In this connection one can easily prove, using ideas similar to those above, the following.

Theorem 1.6. Suppose $T \in \mathcal{P} \mathcal{W}(\mathcal{H})$, the positive-operator-valued function

$$
t \rightarrow(1-t)^{1 / 2}\left(\sum_{j=0}^{\infty} t^{j} T^{* j} T^{j}\right)^{1 / 2}, \quad t \in[\varepsilon, 1), 0<\varepsilon<1,
$$

is decreasing, and the inequalilty (5) holds for some positive number $\alpha$ and a strictly increasing sequence $\left\{t_{k}\right\}_{k \in \mathbb{N}}$ of real numbers converging to 1 . Then $T$ is similar to a contraction.

Another natural question is whether we can weaken the assumption (23) to

$$
\left(1-t_{k}\right) \sum_{j=0}^{\infty} t_{k}^{j} T^{* j} T^{j} \geq \alpha\left(I-P_{\cup_{n=0}^{\infty} k \operatorname{ker}\left(T^{n}\right)}\right),
$$

and preserve the conclusion in Corollary 1.5. The same counterexample of Foguel in [2] shows that there exists an operator satisfying (25) which is not polynomially bounded.

Remark. This paper constitutes part of the author's Ph.D. thesis written at Texas A\&M University under the direction of Professor Carl Pearcy. We thank the referee of this paper who suggested the idea for improving Corollary 1.5.

Added in proof. Vern Paulsen has pointed out that the same techniques employed herein can be used to obtain the stronger result that under the hypotheses of Theorem 1.1 or $1.2, T$ is completely polynomially bounded, and thus is similar to a contraction. 


\section{REFERENCES}

1. A. Lebow, A power-bounded operator that is not polynomially bounded, Michigan Math. J. 15 (1968), 397-399. MR 38:5047

2. S. R. Foguel, A counterexample to a problem of Sz.-Nagy, Proc. Amer. Math. Soc. 13 (1964), 788-790. MR 29:2646

3. B. Sz.-Nagy, On uniformly bounded linear transformations in Hilbert space, Acta Sci. Math. (Szeged) 11 (1947), 152-157. MR 9:191

4. P. R. Halmos, On Foguel's answer to Nagy's question, Proc. Amer. Math. Soc. 13 (1964), 791-793. MR 29:2647

5. P. R. Halmos, Ten problems in Hilbert space, Bull. Amer. Math. Soc. 76 (1970), 887-933. MR 42:5066

Department of Mathematics, Texas A\& M University, College Station, Texas 77843 